\title{
Estimating Observation Error Statistics Using a Robust Filter Method for Data Assimilation
}

\author{
Yue Wang ${ }^{\mathrm{a}}$, Yulong Bai ${ }^{\mathrm{a}, 1}$ \\ ${ }^{a}$ College of Physics and Electrical Engineering, Northwest Normal University, Lanzhou \\ 730070, China
}

\begin{abstract}
For the data assimilation algorithms, the observation error covariance plays an important role, because they control the weight that is given to the model forecast and to the observation in the solution, i.e., the analysis. In order to easily calculate, we often assume observation to be a diagonal matrix, however, the observation errors are correlated to the state and have a certain dependence on time, such as certain observing types which are remotely sensed. In this work, we obtain the time-dependent and correlated observation error by the method of observation error estimation in the data assimilation system. We combine the ensemble time-local H-infinity filter (EnTLHF) with an estimate of observation error covariance matrix, named ensemble time-local H-infinity filter with observation error covariance estimation (EnTLHF-R). In the experiment, a classical nonlinear Lorenz-96 model to evaluate the performance of new method is used. The results show that the robust filtering with observation error estimation is more accurate, more robust, and the filtering is more stable.
\end{abstract}

Keyword: Robust filtering; Observation error covariance; Lorenz-96 model; Robustness

\section{Introduction}

Data assimilation is a statistical process that combines numerical models with systematic observations to estimate the state of spatiotemporal dynamic systems. With the complexity and requirements of the actual situation, Evensen[1] used the idea of ensemble for Kalman filtering, and proposed the ensemble Kalman filter (EnKF) to solve the limitations imposed by dynamic models for complex nonlinear systems, However, the Kalman filtering-based method normally takes the error statistics as Gaussian distributions. For nonlinear, this assumption is often false. To address the issue of non-Gaussian error statistics, robust filtering is used to solve this limitation in the assimilation system[2] .Compared to Kalman filtering, robust filtering can guarantee that the error growth rate is bounded, and does not require certain assumptions about the model and observation system, Luo and Hoteit [3] apply the idea of ensemble to robust filtering to form ensemble time-localized robust filtering(EnTLHF). The observation error in the data assimilation usually includes two

\footnotetext{
${ }^{1}$ Corresponding author address: Yulong Bai, College of Physics and Electrical Engineering, Northwest Normal University, Lanzhou Gansu, 730070, China. E-mail: yulongbai@gmail.com
} 
parts, instrument error and representation error. We often only consider the instrument error and assume it is an unit diagonal array, however recent literatures indicate that representation errors are correlated and have a certain dependence on the time and state [4][5][6]. And recent literatures indicate that observation errors are correlated and have a certain dependence on time and state [7][8][9]. Many scholars [10][11] have demonstrated that a rough approximation of the observed error covariance matrix may also provide significant benefits. And Desroziers et al [12] introduced a method based on two type innovations in the observation space to obtain the estimation of observation error covariance. So we will apply the estimating method into robust filtering and proposed a new method named robust filtering with observation error estimation (EnTLHF-R), and we will estimate observation error by DBCP method, the estimated observation error obtained is time-dependent and is related to the background and state of the previous moment.

In the work, we use typical Lorenz-96 chaotic system to compare the robustness and accuracy of robust filtering method with observation covariance estimation and robust filtering method, the results show considering estimation of observation error in the assimilation could improve accuracy and robustness.

In this paper. Section 2 presents the robust filtering method and how to apply the method of observation error covariance to the robust filtering. In the Section 3, we access the effectiveness of the new method to estimate observation error covariance and show the robustness of robust filtering with observation error covariance estimation. Concluding remarks are followed in summary.

\section{Theoretical Background}

\subsection{Time-local H-infinity Filter (TLHF).}

$\mathrm{HF}[2]$ is one of the robust filters involving the properties of the model and the observation system. Luo and Hoteit[3] applied the objective function in HF to Kalman filtering and proposed a new filtering method named time-localized $H_{\infty}$ filtering (TLHF). The local cost function of TLHF is written as follows:

$$
\begin{aligned}
& J_{x, i}^{H F}=\frac{\left\|x_{i}-x_{i}^{a}\right\|_{S_{i}}^{2}}{\left\|x_{i}-x_{i}^{b}\right\|_{\left(\Delta_{i}^{b}\right)^{-1}}^{2}+\sum_{i=0}^{N}\left\|u_{i}\right\|_{Q_{i}^{-1}}^{2}+\sum_{i=0}^{N}\left\|v_{i}\right\|_{R_{i}^{-1}}^{2}} \\
& \frac{1}{\lambda}>\frac{1}{\lambda^{*}} \equiv \inf _{x_{i}^{a}} \sup _{x_{i}, u_{i}, v_{i}} J_{x, i}^{H F}
\end{aligned}
$$

Where $v_{i}, \quad u_{i}$ represent process noise and measurement noise respectively. The subscript $Q_{i}^{-1},\left(\Delta_{i}^{b}\right)^{-1}, R_{i}^{-1}$ is called the information matrix. $x_{i}$ and $x_{i}^{a}$ are truth and estimating value of system. Equation (2) is the minimum and maximum criterion, where $\sup _{x, i, i_{i}}$ represent the supremum of the cost function $J_{x, i}^{H F}$, relating to variables $x_{i}, u_{i}, v_{i}$ 
$x_{i}, u_{i}, v_{i} ; \inf _{x_{i}^{a}}$ is the infimum responding to $x_{i}^{a} . \lambda^{*}$ ensures that there is a value $\lambda$ that meets the minimum and maximum criteria ${ }^{[2]}$.

Concretely, let $x_{i}^{b}=\left\{x_{i, j}^{b}: x_{i, j}^{b}=M_{i, i-1}\left(x_{i-1, j}^{a}\right), j=1, \cdots, n\right\}$ be the n-member background ensemble, deriving from the prediction of the analysis ensemble $x_{i-1}^{a}=\left\{x_{i-1, j}^{a}, j=1, \cdots, n\right\}$ at the precious cycle.

The prediction step of the EnTLHF:

$$
\begin{aligned}
& \bar{x}_{i}^{b}=\operatorname{mean}\left(x_{i}^{b}\right) \\
& \hat{\Delta}_{i}^{b}=\operatorname{cov}\left(x_{i}^{b}\right)+Q_{i}
\end{aligned}
$$

The filtering step of the EnTLHF:

$$
\begin{aligned}
& \bar{x}_{i}^{a}=\bar{x}_{i}^{b}+G_{i}\left(y_{i}-H_{i}\left(\bar{x}_{i}^{b}\right)\right) \\
& \left(\Delta_{i}^{a}\right)^{-1}=\left(\Delta_{i}^{b}\right)^{-1}+\left(H_{i}\right)^{T}\left(R_{i}\right)^{-1} H_{i}-\lambda_{i} S_{i}=\left(P_{i}^{a}\right)^{-1} \\
& \hat{G}_{i}=\hat{\Delta}_{i}^{a}\left(H_{i}\right)^{T}\left(R_{i}\right)^{-1}
\end{aligned}
$$

Subject to the constraints:

$$
\left(\Delta_{i}^{a}\right)^{-1}=\left(\Delta_{i}^{b}\right)^{-1}+\left(H_{i}\right)^{T}\left(R_{i}\right)^{-1} H_{i}-\lambda_{i} S_{i}=\left(P_{i}^{a}\right)^{-1}-\lambda_{i} S_{i} \geq 0
$$

Here $\Delta_{i}$ represents the uncertainty matrix, similar with the $P_{i}^{a}$ in the $\mathrm{KF}$, and $G_{i}$ is the gain matrix. The symbol " $\geq 0$ " means that the matrix is a semi-positive definite matrix. For Equation (6), the condition satisfying the semi-positive definite matrix is related to the value of variables $R_{i}, \lambda_{i}, S_{i}$. At the same time, we know that the value of $\lambda_{i}$ is related to $R_{i}, Q_{i}, S_{i}$. In Equation (1), the value of $R_{i}, Q_{i}, S_{i}$ is usually chosen freely by the designer[3][13], which motivates us to improve this algorithm in this article.

In Equation (6), we make some formula transformation:

$$
\lambda_{i} S_{i}=c\left[\left(\Delta_{i}^{b}\right)^{-1}+\left(H_{i}\right)^{T}\left(R_{i}\right)^{-1} H_{i}\right]=c\left(p_{i}^{a}\right)^{-1} \quad 0<\mathrm{c}<1
$$

$\mathrm{C}$ is the local performance level, and $S_{i}$ is the information matrix that can be chosen by designer, so we consider the specific term of $\lambda_{i} S_{i}$, building the relation between $\lambda_{i} S_{i}$ and $P_{i}^{a}$. By inflating covariance, so we named an uncertainty inflation technique.

So the analysis error covariance becomes:

$$
\left(\Delta_{i}^{a}\right)^{-1}=(1-c)\left(P_{i}^{a}\right)^{-1}
$$


Where the $c$ called the performance level coefficient, which is related to the $\lambda_{i}$ value.

\subsection{Method of Estimating Observation Error}

The DBCP method is based on the two types of innovation statistics between observations, forecasts and analysis. $d^{b}=y-H\left(x^{f}\right)$ named the background innovation and $d^{a}=y-H\left(x^{a}\right)$ named the analysis innovation. Taking their statistical expectation, then could obtain observation error covariance matrix.

$$
E\left[d^{a}\left(d^{b}\right)^{T}\right] \approx R
$$

\subsection{Observation Error Covariance Matrix}

The observation error includes two parts, instrument error and representation error:

$$
\begin{aligned}
& R^{T}=R^{I}+R^{H}=\sigma_{D}^{2} \varepsilon^{I}+\sigma_{C}^{2} \varepsilon^{H} \\
& \varepsilon^{H}(r)=(1+|r| / L) \exp (-|r| / L)
\end{aligned}
$$

Where $R^{T}$ represents the true observation covariance matrix, $R^{I}$ represents the instrument error covariance matrix, and $R^{H}$ represents the correlated representative error covariance matrix. $\sigma_{D}^{2}$ is the uncorrelated error variance, $\varepsilon^{I}$ is the instrument error, and $\sigma_{C}^{2}$ is the correlated error variance. $\varepsilon^{H}$ is the correlation between two points separated by distance[6].

\subsection{The Robust Filtering with Observation Error Estimation (EnTLHF-R)}

In general, the robust filtering with observation error estimation consist four parts: initialization, running EnTLHF steps, computing innovations and estimating observation. The schematic diagram of robust filtering with observation error estimation is show in Table 1.

Table 1 Description of the ensemble time-local filter with observation error covariance estimation

\begin{tabular}{l}
\hline Algorithm: EnTLHF-R \\
Initialization: Generate initial ensemble members $\left\{x_{0}^{a, i}\right\}, i=1, \cdots, N$,observation vectors $y_{i}$, Initial \\
background covariance matrix $P_{0}^{f}$, Determine the number of DBCP diagnostic samples $N_{s}$, Assume \\
that the initial observation error covariance is $R_{\text {input }}$. \\
For $\mathrm{i}=1$ :assimilation step $\left(i=2 N_{s}\right)$ \\
$\quad$ ENTLHF: $x_{i}^{f}=\left\{x_{i, j}^{f}: x_{i, j}^{f}=M_{j}\left(x_{i-1, j}^{a}\right) \mathrm{j}=1, \ldots, n\right\}$ \\
$\quad$ If $\quad l \leq N_{s}$ \\
$\quad R_{l}=R_{\text {input }}$ \\
\hline
\end{tabular}


else

$$
R_{l+1}=E\left[d^{a}\left(d^{b}\right)^{T}\right]
$$

end if

Prediction step: $\bar{x}_{i}^{b}=\bar{x}_{i}^{f}=\operatorname{mean}\left(x_{i}^{f}\right)$

$$
\Delta_{i}^{b}=\operatorname{cov}\left(x_{i}^{b}\right)+Q_{i}
$$

Obtain background innovation: $d_{i}^{b}=y_{i}-H\left(\bar{x}_{i}^{b}\right)$

Filtering step: $\left[p_{i}^{a}, K_{l}\right]=\operatorname{ETKF}\left(X_{i}^{b}, Q_{i}, H_{i}\right)$

$$
\begin{aligned}
& G_{i}=\left(I_{m}-\lambda_{i} P_{i}^{a} S_{i}\right)^{-1} K_{i} \\
& x_{i}^{a}=\bar{x}_{i}^{b}+G_{i}\left[y_{i}-H\left(\bar{x}_{i}^{b}\right)\right]
\end{aligned}
$$

Obtain analysis innovation: $d_{i}^{a}=y_{i}-H\left(\bar{x}_{i}^{a}\right)$

If $\left(\Delta_{i}^{a}\right)^{-1}=\left(p_{i}^{a}\right)^{-1}-\lambda_{i} S_{i} \geq 0$

Return $\lambda_{i}$

Else

End for

\section{Numerical Experiment}

\subsection{The Lorenz-96 Model.}

The Lorenz-96 model is defined by ordinary differential equations (ODEs) defined over a periodic domain of variables indexed by $\mathrm{n}=0 \ldots N_{x}-1$ where $N_{x}=40$

$$
\frac{d x_{n}}{d t}=\left(x_{n+1}-x_{n-2}\right) x_{n-1}-x_{n}+F
$$

This model is an idealized for state estimation problems. For computational stability, a time step of 0.05 units is adopted. In the experiment, the value of the forcing term $F$ can be changed, representing different degrees of model error and the solution of the Lorenz-96 equation is obtained by using the classic fourth-order Runge-Kutta. The total length of the iteration is 1500 steps.

\subsection{Performance Comparison of two Robust Filtering Methods.}

In this section, we show the performance of traditional robust filtering (EnTLHF) and new method (EnTLHF-R) on the Lorenz96 model. The results show the RMSE mean of EnTLHF and EnTLHF-R when observation error is RT. 

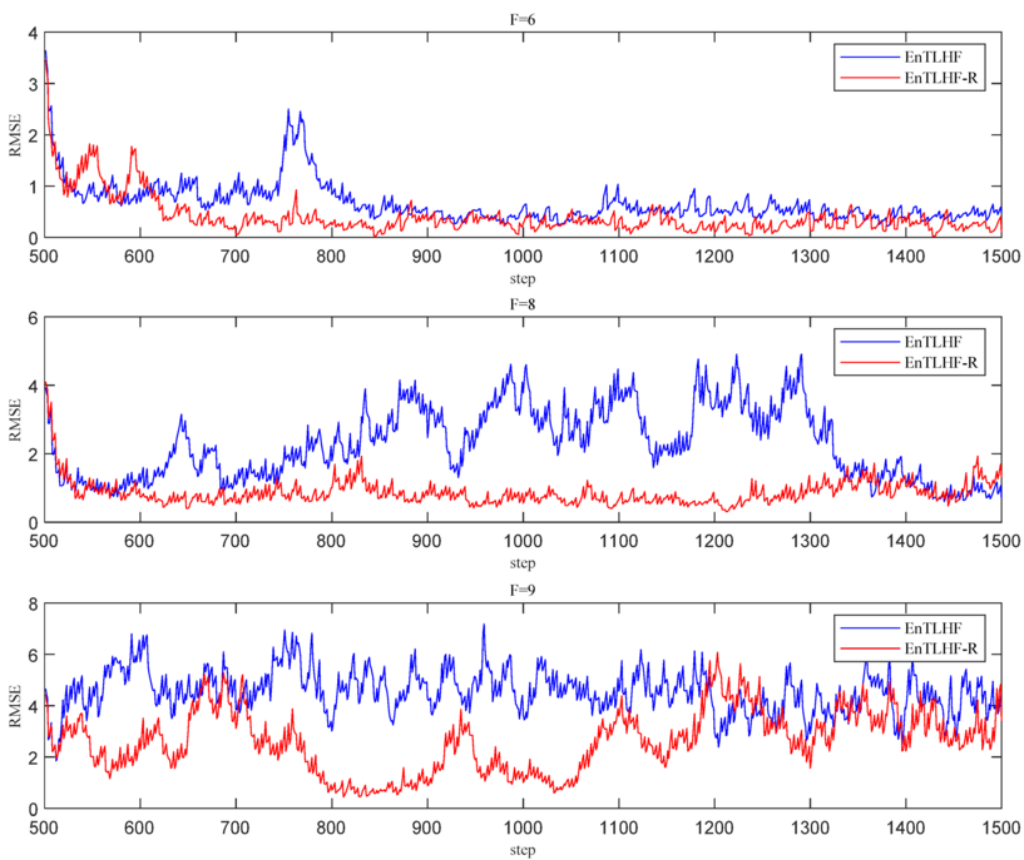

Figure 1. When forcing parameter $F=6,8,9$, the RMSE mean of EnTLHF and EnTLHF-R algorithm in the Lorenz-96 model

The results show: (1) the robust filtering with observation error estimation (EnTLHF-R) has lower Root Mean Squared Errors (RMSE) when the force parameter changes. (2)As force parameter increases the model error increase correspondingly, the RMSE of EnTLHF and EnTLHF-R increase, however the RMSE of EnTLHF-R is always lower EnTLHF, The larger the forcing parameter is, the more obvious the effect will be. Overall, the robust filtering with observation error estimation (EnTLHF-R) is more robust when model error is changing; this indicates that the new method improves the performance; the estimation of observation error covariance in the data assimilation may provide benefit.

\subsection{Estimation of Observation Error Covariance}

In this section, we access the ability of new method to accurately estimate R. In the experiment, we set the $R T$ as the reference observation error covariance. The above Figure 2 (a) represents the covariance of the observation error obtained in the first estimation (the 126th assimilation), and the figure 2 (b) represents the estimated covariance of the observation error obtained in the 250th assimilation. 


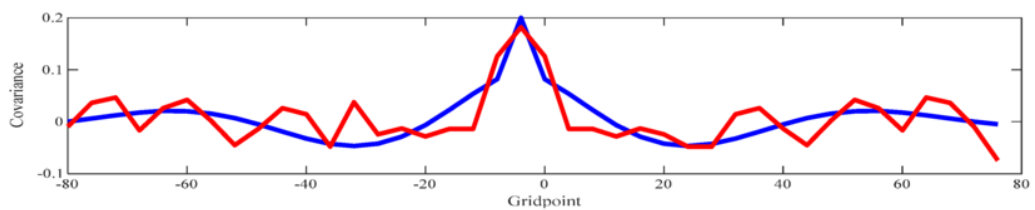

(a)

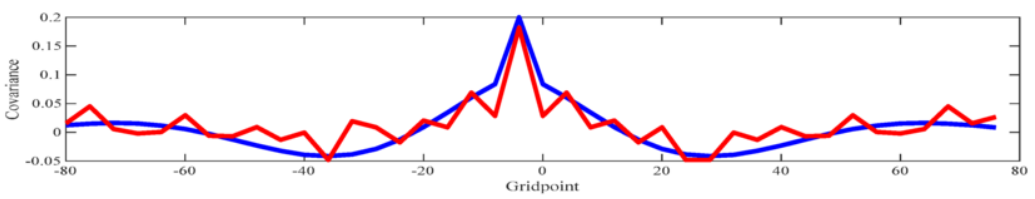

(b)

Figure 2. The blue line represents the true covariance matrix, and the red line represents the estimated covariance matrix. (a) Obtaining R using the first 125 innovations. (b) Obtaining covariance using the last 125 innovations

The results show: (1) when taking into account the spatial error correlations, the Desroziers diagnostic is useful to estimate off-diagonal term of observation. (2)The last estimating of $\mathrm{R}$ is more fitting RT; it also suggests that estimating method could to obtain a time-dependent estimate of correlate observation.

\section{Summary}

This paper introduces traditional robust filtering method and new robust filtering method. In particular, the diagnostic method is coupled to obtain the time-varying observation error, in the simulation experiments; we compare the performance of robust filtering with observation error estimation and original robust filtering with Lorenz-96 model. In addition, we access the observation error estimation method; the results prove taking correlated observation into account in data assimilation is more benefit.

\section{Acknowledgement}

This work is funded by the NSFC (National Natural Science Foundation of China) project (grant number: 41861047 and 41461078), also based on Northwest Normal University young teachers' scientific research capability upgrading program (NWNU-LKQN-17-6).

\section{References}

[1] Evensen G. Sequential data assimilation with a nonlinear quasi-geostrophic model using Monte Carlo methods to forecast error statistics, Journal of Geological Research 1994.99:10143-10162

[2] Simon, D. Optimal State Estimation: Kalman, H Infinity, and Nonlinear Approaches. John Wiley \& Sons, Inc.: Hoboken, New Jersey and Canada; 2006.pp 333- 365

[3] Luo XD, Hoteit I. Robust ensemble filtering and its relation to covariance inflation in the ensemble Kalman filter. Month Weather Review. 2011 Jul; 139: 3938- 3953.

[4] Stewart LM. Correlated Observation Errors in Data Assimilation. PhD Thesis, University of Reading.2010 
[5] Weston P. Progress towards the implementation of correlated observation errors in 4d-var. Technical report, Met Office, UK. Forecasting Research Technical Report.2011; 560.

[6] Waller JA, Dance SL, Lawless AS, and Nichols NK. (2014) Estimating correlated observation error statistics using an ensemble transform Kalman filter. Tellus A: Dynamic Meteorology and Oceanography.2014 Set; 66:1, 23294

[7] Janjic and Cohn SE. Treatment of observation error due to unresolved scales in atmospheric data assimilation. Month Weather Review, 2006 Feb; 134: 2900-2915.

[8] Bormann N and Bauer P. Estimates of spatial and inter channel observation-error characteristics for current sounder radiances for numerical weather prediction. I: methods and application to ATOVS data. Quarterly Journal of Royal Meteorological Society. 2010 Apr; 136, 1036-1050.

[9] Liu W. State estimation for discrete-time Markov jump linear systems with time-correlated measurement noise. Automatica. 2017, 76:266-276.

[10] Stewart LM, Dance SL, and Nichols NK. Data assimilation with correlated observation errors: experiments with a 1-D shallow water model. Tellus A. Dynamic Meteorology and Oceanography. 2013 Apr; 65:1, 9546.

[11] Healy SB, and White AA. Use of discrete Fourier transforms in the 1D-Var retrieval problem. Quarterly Journal of Royal Meteorological Society.2005; 131, 63-72

[12] Desroziers G, Berre L, Chapnik B. and Poli P. Diagnosis of observation, background and analysis-error statistics in observation space. Quarterly Journal of Royal Meteorological Society. 2005 Oct; 131: 3385-3396

[13] Bai YL, Zhang ZH. Inflating transform matrices to mitigate assimilation errors with robust filtering based ensemble Kalman filters. Atmospheric science letters.2016 Jul; 17: 470-478. 